\section{Laserscanmikroskopie erleichtert Diagnostik bei Verdacht auf ein Basalzellkarzinom}

\author{
In der Hand eines damit vertrauten Dermatologen lässt sich mit der konfokalen \\ Laserscanmikroskopie das Basalzellkarzinom so gut wie durch die Beurteilung \\ nach einer Stanzbiopsie diagnostizieren. Das gilt auch für die Subtypisierung.
}

W ie zuverlässig sich die konfokale Mikroskopie im Vergleich zur Stanzbiopsie in der Diagnostik des Basalzellkarzinoms nutzen lässt, haben Dermatologen aus den Niederlanden in einer prospektiven Kohortenstudie mit 100 Patienten an zwei Zentren der Maximalversorgung in Amsterdam überprüft. Sie verwendeten dazu zwei identische Mikroskope des Typs VivaScope $1500^{\circledR}$ (Mavig GmbH). Durch die Auflichttechnik wird bei diesem Mikroskop die Haut mit einem Punktstrahllaser (830-nm-Diodenlaser, maximal $30 \mathrm{~mW}$ ) fokussiert beleuchtet. Die Eindringtiefe ist auf etwa $250 \mu \mathrm{m}$ begrenzt.

Die Patienten wurden randomisiert zwei Gruppen zugeordnet. In der einen Gruppe wurden Diagnose und Subtypisierung eines Basalzellkarzinoms mithilfe der Mikroskopie und danach eine chirurgische Exzision vorgenommen. In der Vergleichsgruppe erfolgte eine geplante Exzision auf Basis der histologischen Untersuchung und Subtypisierung des Stanzbiopsats. Primärer Endpunkt der Untersuchung war die Übereinstimmung zwischen den beiden Diagnoseverfahren im Vergleich zum Standardverfahren der chirurgischen Exzision. Etwa $43 \%$ der Studienteilnehmer hatten ein superfizielles Basalzellkarzinom, $43 \%$ und 52\% (im Standardarm der Studie) ein noduläres und $14 \%$ der Patienten mit mikroskopischer Diagnose und $6 \%$ der Patienten im Standardarm ein aggressives Basaliom.

Die Sensitivität beim Nachweis eines Basalzellkarzinoms war per Mikroskopie ähnlich hoch wie unter Verwendung einer Stanzbiopsie (100\% versus 93,9\%). Allerdings war die Spezifität (Gesunde korrekt als gesund erkannt) der Diagnostik per Stanzbiopsie deutlich höher
(79\% versus $38 \%$ ). Dabei war das Diagnoseergebnis bei beiden mikroskopierenden Dermatologen mit einer Sensitivität von $100 \%$ und einer Spezifität von $75 \%$ gleich gut. Wenn es um die Identifizierung des aggressivsten Basalzellsubtyps ging, reichte die Übereinstimmungsrate zwischen Diagnostik per Mikroskopie und Exzisionsprobe von 50\% bis $85 \%$ im Vergleich zu $77 \%$ bei der Stanzbiopsie.

Fazit: Der Vorteil der konfokalen Laserscanmikroskopie liegt nach Ansicht der Dermatologen darin, dass das nicht invasiv erreichte diagnostische Ergebnis sofort verfügbar ist und eine Behandlung frühzeitig begonnen werden kann. Stanzbiopsien hingegen seien schmerzhaft und die Diagnose danach nicht sofort verfügbar. Sie halten es für möglich, dass die Mikroskopie die Stanzbiopsie in der Diagnostik und Subtypisierung des Basalzellkarzinoms ersetzen kann. Für eine Entscheidung müssten jedoch noch die Ergebnisse großer prospektiver Studien abgewartet werden. Peter Leiner

Kadouch DJ et al. Diagnostic accuracy of confocal microscopy imaging vs. punch biopsy for diagnosing and subtyping basal cell carcinoma. J Eur Acad Dermatol Venereol 2017; 31: 1641-8

\title{
Sweet-Syndrom bei rheumatoider Arthritis
}

Eine 52-jährige Frau mit rheumatoider Arthritis hatte vor einem Monat erstmals mehrere schmerzhafte, rote Flecken an beiden Handflächen und mehreren Fingern bemerkt. Seit drei Jahren bestand eine Dauertherapie mit Sulfasalazin und Azathioprin, vor eineinhalb Jahren war sie über sechs Monate hinweg mit Etanercept behandelt worden. Zusätzlich zu den Hautveränderungen ergab die Untersuchung typische Veränderungen einer fortgeschrittenen rheumatoiden Arthritis wie eine Ulnardeviation der Langfinger und eine Z-Deformität beider Daumen. Es bestand kein Fieber. Die BKS war mit $50 \mathrm{~mm} / \mathrm{h}$ deutlich erhöht, der CRP-Wert lag bei 20,2 mg/l (Normalwert <3,5), die Leukozytenzahl betrug $3.670 / \mu \mathrm{l}$ mit $87 \%$ Neutrophilen.
In der Hautbiopsie zeigte sich ein papilläres Hautödem mit perivaskulären und interstitiellen lympohistiozytären und neutrophilen Infiltraten, die mit einer akuten febrilen neutrophilen Dermatose, einem Sweet-Syndrom, vereinbar waren. Differenzialdiagnostisch wären in diesem Fall eine Vaskulitis, ein Arzneimittelexanthem oder Hautinfektionen zu erwägen. Nach einer kurzen Stoßtherapie mit Prednisolon bildeten sich die Hautveränderungen rasch zurück. Auch im Verlauf des nachfolgenden Jahres traten sie nicht mehr auf. Das Behandlungsregime der rheumatoiden Arthritis blieb unverändert. Prof. Hermann S. FüeßI

Huang $\mathrm{ZH}$ et al. Sweet's syndrome in a patient with rheumatoid arthritis. N Engl J Med 2017; 377: 769

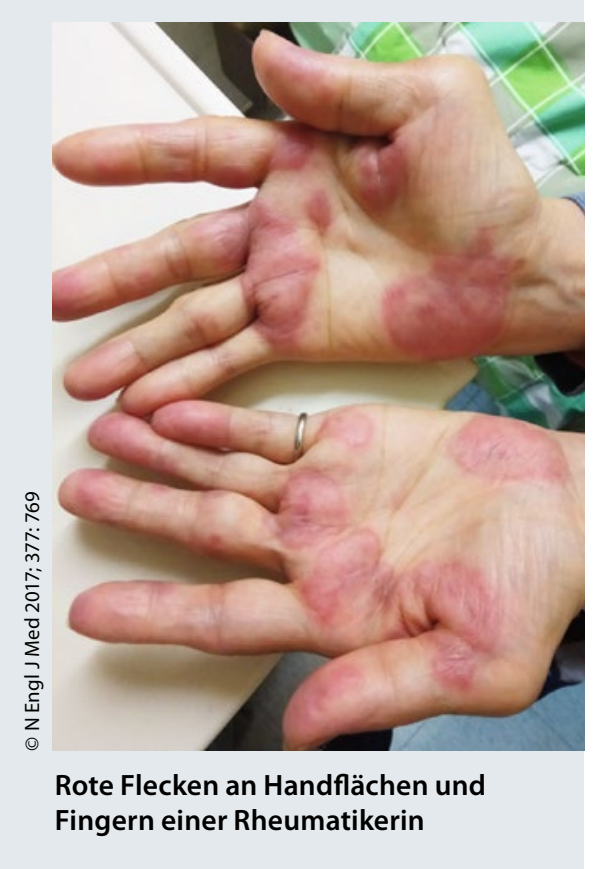

